\title{
UNDERGRADUATE RESEARCH STUDENT PERCEPTIONS
}

\begin{abstract}
Health professionals are required to generate evidence via research in order to validate their practice. Undergraduate students in the professions allied to medicine complete a research component in their final year of study. The assumption is that this component will equip them with the knowledge, skills and attitudes to undertake research as

DAWSON D, BSC'; FAURE M, M.Phil'; JULIUS B, BSC(HONS)'

' Physiotherapy Department, University of Stellenbosch clinicians. In this retrospective study, a questionnaire was used to examine the perceptions and experiences of students relating to their research projects. The study population was students studying in the professions allied to medicine on the Tygerberg Campus of the University of Stellenbosch.

The response rate was $77 \%(n=106)$. Of these students, $69 \%$ enjoyed implementing their research project, whilst $28 \%$ did not. However, physiotherapy students experienced this component of their course most negatively. The most positive perceptions of the students related to educational benefits and interest, whilst the most negative results related to time restraints and stress.

In planning for the future, it is important to evaluate the outcomes of the research component of the undergraduate curriculum and its possible effects on the development and practice of the physiotherapy profession.
\end{abstract}

\section{KEY WORDS: UNDERGRADUATE RESEARCH; STUDENT PERCEPTIONS; PHYSIOTHERAPY; EVIDENCE}

\section{INTRODUCTION}

Physiotherapists are becoming increasingly accountable to the client, the medical aid societies paying for services and the public at large. The need for increased accountability has accelerated interest in generating appropriate and acceptable evidence for endorsing many areas of clinical practice (Law and Baum 1998). This can only be achieved through evidence-based practice, which in turn is grounded in appropriate and valid research.

However, it has been suggested that no body of evidence exists at present to prove that the overwhelming majority of physiotherapeutic clinical practice techniques produces the best effect (Newham 1997; Thomson-O'Brien and Moreland 1998).

A requirement of the degree curriculum for physiotherapists and many other health care professionals, is that students undertake research under the supervision of lecturers, individually or in groups. The culmination of this research project is a written and/or verbal presentation.

In August each year the Tygerberg Campus of the University of Stellenbosch hosts two academic year days. One is a Faculty Academic Year Day (FAYD) where lecturers and clinicians attached to the Faculty present their research findings. The other is a Student Academic Year Day (SAYD) where selected students present the results of their research projects to students and staff of the Faculty. It has been observed that student attendance at both of these occassions is poor. This is in spite of the Faculty policy of freeing students from lectures and clinical responsibilities in order to attend these presentations. In order to examine the student attendance and perceptions of these two occasions a questionnaire was distributed to all students in the Medical Faculty. This questionnaire was a convenient vehicle in which to question the students' attitudes towards, and subjective experiences of, their own research projects.

\section{METHODS AND MATERIALS}

A retrospective study was done making use of a questionnaire that was compiled by three researchers from the Department of Physiotherapy in consultation with one person from the Department of Community Health and one person from the Department of Consumer Studies. The questionnaire was divided into four sections, and included both qualitative and quantitative questions relating to:
- personal information, including year of study and department

- attendance at both of the academic year days

- students' perceptions of various aspects of both of the academic year days

- the students' experiences and perceptions of their own research projects

The questionnaire was distributed and collected by the class representatives of each class on two occasions:

- at the end of 1998 to all final year students from the professions allied to medicine and fifth year medical and dental students;

- early in 1999 to all students attending lectures on campus, except the second year students who had just arrived on the Medical Campus and would not yet have had the opportunity of attending the academic year days.

\section{CORRESPONDENCE TO:}

D Dawson

Department of Physiotherapy,

Faculty of Medicine,

PO Box 19063,

Tygerberg

7505

Tel: (021) 938-9300 (W) 
The study population therefore included all other students from the departments of physiotherapy (PT), occupational therapy (OT), speech therapy (SpT), human nutrition (HN), nursing $(\mathrm{N})$, medicine $(\mathrm{M})$ and dentistry (D) who attended classes on the Tygerberg campus during 1998. This led to the exclusion of the sixth year medical and dental students, as they spend most of their time in clinical activities away from the Tygerberg campus.

A research project, completed early in the second semester of their fourth year, is a compulsory component of the curriculum for students from the professions allied to medicine, although an optional component in the medical and dental curriculum. This often results in predominantly those medical and dental students who are interested in research following this option. Therefore, for the purposes of this article, only that information relating to the research experiences and perceptions of fourth year students of 1998 in the professions allied to medicine $(n=106)$, will be presented and discussed. Special reference to physiotherapy students will be made where appropriate.

Qualitative responses initially were coded independently by two of the researchers into categories of positive and negative attitudes. As comments could easily be placed in either of these broad categories, $100 \%$ agreement on the initial coding was reached. These categories were then further refined by the same two reseachers into subcategories of themes in accordance with qualitative methodology. Consensus was then reached by all three of the researchers on the final themes which are documented in Table 2.

\section{RESULTS}

The total response rate was $42 \%$ $(n=588)$. The response rate of the fourth year students in the departments allied to medicine are given in Table 1 . The students' enjoyment of research, or lack thereof, is shown in Figure 1. However, as can be seen from Figure 2, the physiotherapy students of 1998 were more negative towards their research projects than students from other departments.

TABLE 1. Total response rate per department

\begin{tabular}{|l|c|c|c|}
\hline \multicolumn{1}{|c|}{1998} & $\begin{array}{c}\text { Total Nos } \\
\text { in Class }\end{array}$ & $\begin{array}{c}\text { Total No of } \\
\text { Responses }\end{array}$ & $\begin{array}{c}\% \text { Response } \\
\text { Rate }\end{array}$ \\
\hline $\begin{array}{l}\text { Physiotherapy } \\
\text { (PT) }\end{array}$ & 44 & 37 & 84 \\
\hline $\begin{array}{l}\text { Occupational Therapy } \\
\text { (OT) }\end{array}$ & 28 & 26 & 93 \\
\hline $\begin{array}{l}\text { Nursing } \\
\text { (N) }\end{array}$ & 27 & 19 & 70 \\
\hline $\begin{array}{l}\text { Human Nutrition } \\
\text { (HN) }\end{array}$ & 25 & 16 & 64 \\
\hline $\begin{array}{l}\text { Speech Therapy } \\
\text { (SpT) }\end{array}$ & 137 & 106 & 62 \\
\hline TOTAL & & 87 \\
\hline
\end{tabular}

TABLE 2. Numbers of physiotherapy responses compared to total

\begin{tabular}{|l|r|r|l|r|r|}
\hline \multicolumn{1}{|c|}{ POSITIVE } & Total & PT & \multicolumn{1}{|c|}{ NEGATIVE } & Total & PT \\
\hline Interesting topic & 16 & 3 & Uninteresting topic & 2 & 0 \\
\hline Learnt a lot/educational & 29 & 4 & Stressful/pressured & 6 & 2 \\
\hline Work in groups & 7 & 0 & Work in groups & 2 & 0 \\
\hline Good guidance/ support & 2 & 1 & Poor guidance/ support & 2 & 0 \\
\hline Stimulating/interesting & 29 & 3 & Too few credits & 3 & 3 \\
\hline Contribute to knowledge & 6 & 2 & Time consuming/lack & 24 & 11 \\
\hline Enjoy research & 7 & 1 & Dislike research & 5 & 3 \\
\hline Other & 16 & 4 & Other & 9 & 4 \\
\hline
\end{tabular}

TABLE 3. Strongest trends in perceptions

\begin{tabular}{|l|c|c|c|c|c|}
\hline \multicolumn{1}{|c|}{ POSITIVE } & $\begin{array}{c}\text { Nos } \\
\text { of PT }\end{array}$ & $\begin{array}{c}\text { Nos } \\
\text { of HN }\end{array}$ & NEGATIVE & $\begin{array}{c}\text { Nos } \\
\text { of PT }\end{array}$ & $\begin{array}{c}\text { Nos } \\
\text { of HN }\end{array}$ \\
\hline Learnt a lot/ educational & 4 & 11 & Time consuming/lack & 11 & 1 \\
\hline Stimulating/ interesting & 3 & 9 & & & \\
\hline Interesting topic & 3 & 5 & & & \\
\hline
\end{tabular}

The responses of the physiotherapy students in relation to all of the other students concerning the positive and negative themes are outlined in Table 2. The majority of the positive responses suggested that they had learnt a lot, and that it had been an interesting and stimulating experience. Time issues were the overwhelming negative responses, while ambivalence for research per se and its importance for practice was implied.

Physiotherapy students and those students from the Department of Human Nutrition differed most strongly in their perceptions of their research projects. The main differences are shown in Table 3. This table also highlights the possible relationship between stress and time constraints and the way that students experienced these research projects.

Of the 37 physiotherapy students who responded to the questionnaire, $32 \mathrm{did}$ not attend the research presentations at SAYD. Of these, eight students indicated that they did not attend due to their workloads, ten students blamed too many academic deadlines or tasks and three students indicated that they had a previous appointment.

In reply to the question, "How did you spend your class free time on Faculty Academic Year Day?" twelve students responded that they had spent part of the time relaxing or sleeping, thirteen others had spent time studying or working on 
FIGURE 1. Enjoyment of research project

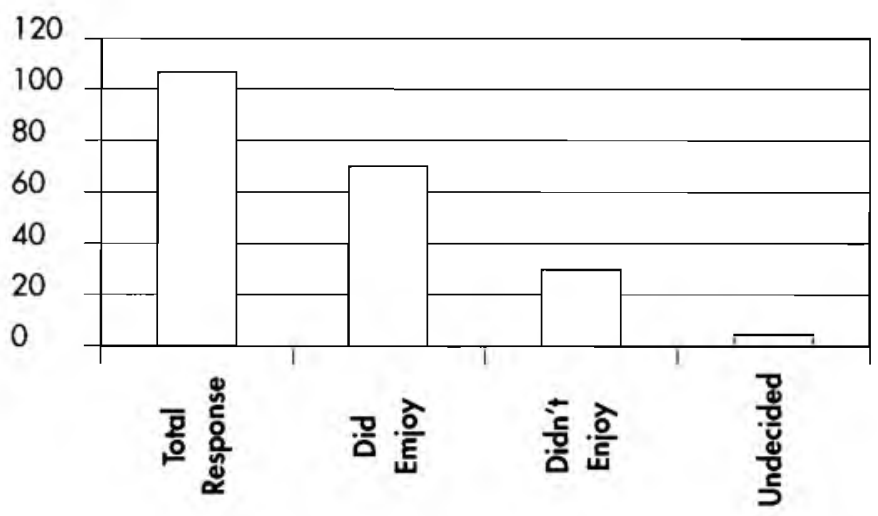

FIGURE 2. Departmental responses - enjoyment

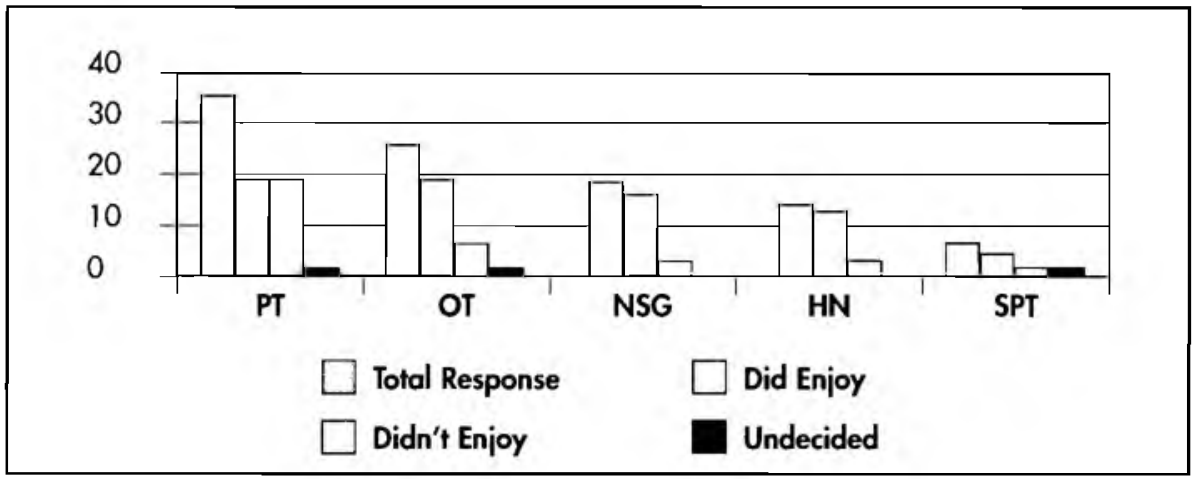

academic tasks while fourteen had had clinical demands. $1 n$ responding to the question "What would motivate you to attend the presentations?" ten physiotherapy students said a lighter workload, and only three said a greater interest in research.

\section{DISCUSSION}

In Figure 1 the responses of students suggest that, in total, more students did enjoy the research project than those who didn't. However, in Figure 2 it can be seen that a significant number of physiotherapy students responded that they had not enjoyed this component. Feedback from qualitative questions indicated that pressures associated with time constraints contributed strongly to the lack of enjoyment which students from most departments experienced. Comments such as "I enjoyed the research but not enough time", and "... had to be rushed", were made by a number of students. On the other hand, the Human Nutrition students were the most positive towards their projects, with the time factor being mentioned least often (Table 3). The research protocol is completed by the end of the third year of study in this department. These students are then given a six week "block" to work fulltime on collecting and analysing their data at the beginning of their fourth year. For the next few months the students are not given "class time" to complete their projects, but must do so in their own time. In contrast, the physiotherapy students were only given one afternoon per week for six months of their fourth year to work on their projects. It is obviously more difficult to work in such a "stop/start" manner. Interestingly, the lack of credits which might be translated as lack of recognition for the amount of work done or, more seriously, as a lack of recognition for the importance of the research module, was of concern to physiotherapy students.

The underlying rationale for including the research component into the undergraduate curriculum, is that having had first hand experience of the research process, students will develop research skills and a questioning attitude to practhat as graduates, physiotherapists - and other health care clinicians - will utilise tice and to the literature. It is assumed these attributes to evaluate their com petence and to justify their practice as clinicians.

Whether these aims are being achieved as efficiently and effectively as possible is questioned by many (Kitchen 1999; Summers et al 1998). In motivating their innovations for a research curriculum for medical residents, Summers et al (1998) state that “... even residents who are academically minded can be dissuaded by the seeming drudgery of scientific inquiry." They suggest that one reason for this reluctance is the notion that the research requirement is considered to be only another hurdle in the goal of the completion of their residency. The notion of the drudgery associated with a research project "killing" enthusiasm for research in physiotherapy students, was also raised by Kitchen (1999). If this process, central to professional practice is considered by undergraduates to be only another hurdle to get over, the question to be asked then is whether there is much chance that, as clinicians, research will be afforded the status that is essential for the development of effective, evidence-based practice?

One therefore needs to examine whether the process of research and its product, whether a written or oral presentation, achieves its purpose and thereby justifies its inclusion in the undergraduate curriculum as merely an isolated and self-fulfilling module. Is it not more important that the "research" process is both integrated, and central, to an inquiring and rigorous approach to professional practice which should be fostered in undergraduate - and postgraduate - students throughout their courses? In this way it might be more likely that the research process will become an integral part of everyday professional practice.

The Research and Development Group (RDG) of the Chartered Society of Physiotherapy (1996) state that the concept of research focusses on the concept of evaluation as understood in clinical physiotherapy. Awareness of a question that needs to be answered or a problem that needs to be addressed, collection and critical evaluation of information, formulation of the problem, intervention, documentation, re-evaluation and 
reflection are all skills both of clinical decision making and research. Both processes are thus closely related. By integrating research skills into clinical skills in order to enhance evidence-based practice and the methods of inquiry used in each, a solid foundation can be laid for integrating practice and research.

Moore (1997) raises the need for the development of a strong culture of research in physiotherapy. She maintains that without such a culture, physiotherapy can be "nothing more than an art, though like medicine and law it will always be partially an art". One way of contributing to, and keeping up to date with professional developments is achieved by critically reading the literature, applying research principles in interpreting the results and implementing these findings in clinical practice, where appropriate.

A further method of contributing to the development of a profession, is by undertaking research projects and participating in congresses, research presentations and other academic meetings. Newham (1997) stresses the importance of attending meetings at which research findings are presented, in order to implement relevant findings into practice. However, only five out of 37 physiotherapy students attended the academic presentations at the university, with their reasons for not attending reflected by factors such as their workload and time pressures as has already been discussed. Students in all of the departments in the Faculty of Medicine indicated that more encouragement from lecturers would motivate them to attend the presentations.

The primary purpose of academic activities and projects at undergraduate level, is to educate students (Kitchen 1999), and "education" implies a change in behaviour or attitude. Although many students felt that the research component was a "good experience" and felt satisfaction in acquiring new skills only three out of the total number of $106 \mathrm{stu}$ dents related the importance of research to the profession, with comments such as "research is vital for the advancement of any profession".

Do the majority of students really see this component as only the academic "hurdle" suggested by Summers et al (1998) Barriers to research, and the enjoyment thereof are not peculiar to our students. Nolan et al (1994) in Law and Baum (1998) included the lack of time as well as a lack of research skills as being detrimental to the research process for clinicians. A lack of funding, as well as service pressures, which also can be linked to a lack of time, were amongst the barriers identified by Newham (1997), while Thomson-O'Brien and Moreland (1998) included problems with research methods and applicability of studies. It therefore appears that in the last 20 years very little progress seems to have been made in addressing these difficulties, as Balin et al (1980) include a "lack of time and funds" as well as a "lack of familiarity with the research process" at the top of a list of barriers to research!

Unless due care is taken, our profession will not develop an evidence-based character. It might be in developing and integrating an inquiry-based approach into all of the components of theory and practice included throughout all four years of the undergraduate curriculum, that both the skills of, and positive attitudes towards, research, might be fostered. Clearly, choices have to be made about "core" knowledge and skills not only for treating clients, but also for the continuation of the profession.

Millson (2000) suggests that we may not only lose physiotherapists to other professional fields because of disillusionment but the loss of elements of our role to other disciplines might accelerate. Most importantly, we might risk the dissatisfaction of patients, other professionals and medical aids because of our failure to provide evidence based practice and capitalise on efficiency (Moore 1997). The expansion of our professional knowledge base is of vital importance in physiotherapy. The responsibility for this task rests with each and every member of the profession (Higgs et al 1998). However, it is the responsibility of the universities to ensure that at the end of their undergraduate training, physiotherapists are equipped not only with a range of technical skills but also with the knowledge and attitudes which might ensure the survival of our profession (Hunt et al 1998).

Increased discussion between all interested parties, formally and infor- mally, needs to take place in order to share experiences and provide a focus for educational innovation at undergraduate and post-graduate levels. If such collegiality is achieved through clinical special interest groups, surely more interaction needs to take place at all levels of the lecturing fraternity too. We must continue to develop strategies that will increase our students' involvement in and enjoyment of research not only so that it can be integrated into all aspects of professional practice, but also to ensure optimum development of our profession. Only then maybe we can fulfil our primary goal of providing the highest quality of care to achieve the best outcomes for our clients in a manner which is cost effective (Summer 1998).

\section{REFERENCES}

Balin A J, Breslin W H, Weirengen K A S, Shepard K F 1980 Research in physiotherapy philosophy: Barriers to involvement and use amongst Californian physiotherapists. Physical Therapy 60: 888-895

Higgs J, Titchen A 1998 Research and Knowledge. Physiotherapy 84(2): $72-79$

Hunt A, Higgs J, Adamson B, Harris L 1998 University Education and the Physiotherapy Professional. Physiotherapy 84(6): 254-264

Kitchen S S 1999 Editorial - Are student research projects good for research and practice? Physiotherapy Research International 4(3): iv-vi

Law M, Baum C 1998 Evidence-Based Occupational Therapy. Canadian Journal of Occupational Therapy 65(3): | 31- | 35

Millson H 2000 Letter to the Editor. The South African Journal of Physiotherapy 56 (1): 28

Moore A P 1997 Research, the Collaborative Approach: Clinicians and academics. Physiotherapy 83(5): 229-234

Newham D J 1997 Physiotherapy for Best Effect. Physiotherapy 83(1): 5-I1

Summers R L, Woodward L H, Sanders D Y, Galli R L 1998 Research curriculum for residents based on the structure of the scientific method. Medical Teacher 20 (1): 35-37

The Research and Development Group 1996 Physiotherapy Research and Continuing Professional Development: The way forwards. Physiotherapy 82(9)

Thomson-O'Brien L, Moreland A 1998 Evidence-based Practice Information Circle. Physiotherapy Canada 50(3): 184-189 